\title{
Energy Efficient Power Aware Multipath Dynamic Source Routing
}

\author{
Kavita Sharma \\ Research Scholar \\ Computer science and engg. JMIT Radaur \\ Kurukshetra University Haryana, India
}

\author{
Vivek Sharma \\ Assistant Professor \& HOD \\ Computer science and engg. JMIT Radaur \\ Kurukshetra University Haryana, India
}

\begin{abstract}
Mobile Ad-hoc Networks (MANET) are consisting of nodes that have limited battery power so the energy efficiency is one of the primary metrics of interest. Energy efficient routing is a major issue in MANET. This paper proposed an Energy efficient power aware multipath dynamic source routing protocol by modifying one of the most popular routing protocols that is Dynamic Source Routing (DSR) protocol which is not at all concerned about power consumption. The proposed Dynamic source routing-Power Aware routing DSRPSR uses the basic concept of traditional DSR and implements energy efficient routing through which it not only enhances the life time of the network but also increases the overall performance of the networks.
\end{abstract}

\section{General Terms}

Energy Efficient algorithm for Dynamic source routing protocol.

\section{Keywords}

Dynamic source routing protocol, DSR, energy efficient routing, Energy efficient dynamic source routing.

\section{INTRODUCTION}

Ad hoc networks are the autonomous systems consist of mobile nodes that communicate with each other using wireless communication. Here a node can be a PDA, a laptop, a mobile phone or another communication device with some characteristics that are limited storage capacity, limited bandwidth and ,limited battery power. An ad hoc network typically refers to any set of networks where all devices have equal status on a network and are free to associate with any other ad hoc network devices in link range.[1][2]

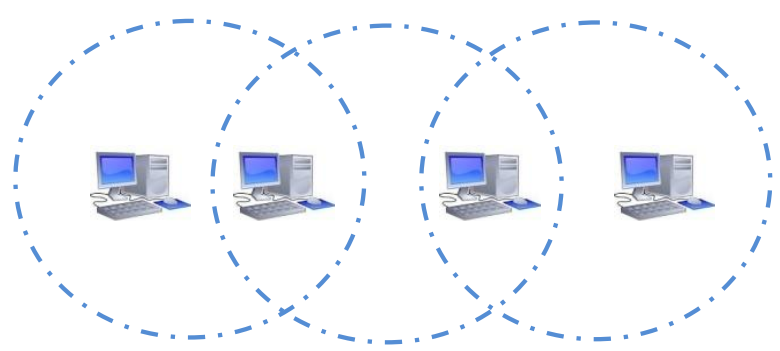

Fig 1 Ad hoc network with four nodes

Ad hoc network do not have any pre-existing infrastructure. They are self-organized, self-configured, and self-controlled networks. This type of network can be set up or deployed anywhere and anytime because it poses very simple setup and no or minimal central administration. The network is characterized by the absence of central administration devices such as base stations or access points. Furthermore, nodes are free to move in any direction, and therefore will change its links to other devices frequently. The primary challenge in building a wireless ad hoc network is to equipping each device to continuously maintain the information required to properly route traffic. This means if link breakages occur the network has to stay operational by building new routes.

\section{DESCRIPTION OF SELECTED ROUTING PROTOCOL}

Mobile networks have attracted significant interest in recent years because of their improved flexibility and reduced costs.

Compared to wired networks, mobile networks have unique characteristics like frequent network topology changes, varying link capacity because of the impacts from transmission power, receiver sensitivity, noise, fading, and interference. Additionally, wireless mobile networks have a high error rate, power restrictions, and bandwidth limitations [1]. Routing is the process of selecting paths in a network along which network traffic can be sent. [2] In a mobile ad hoc network arbitrarily motion of nodes results in unpredictable and frequent topology changes. Additionally, since nodes in a mobile ad hoc network normally have limited transmission ranges, nodes cannot communicate directly with each other. Hence, routing paths in mobile ad hoc networks contain multiple hops, and each node in mobile ad hoc networks has the responsibility to act as a router. Because of the importance of routing protocols in dynamic multihop networks, a lot of mobile ad hoc network routing protocols have been proposed in the last few years. Routing protocols of MANETs fall into two main categories. First are Proactive protocols which are also known as table driven routing protocol in which every node periodically exchange the routing information and maintain network topology information in form of tables.

Nodes continuously evaluate routes by flooding routing information across the network to all other reachable nodes and attempt to maintain up-to-date and consistent routing information. Therefore, a source node immediately gets a routing path when it needs one. On the contrary, reactive protocols build paths on-demand and Dynamic Source Routing (DSR) is an on demand routing protocol. The routing protocols that fall under this category do not maintain the network topology information. They obtain the necessary path when required. Hence they do not periodically exchange any routing information. [6] A route discovery operation invokes a route determination procedure. This discovery procedure terminates either when a route has been found or no route is available after examination for all route permutations. In a mobile ad hoc network, active routes may be disconnected 
due to node mobility. Therefore, route maintenance is an important operation of reactive routing protocols [1]. So DSR employs flooding to discover paths (Section 2.1). Then, to manage situations in which current routes are broken, DSR implements a separate route maintenance procedure (Section 2.2).

An easy way to comply with the conference paper formatting requirements is to use this document as a template and simply type your text into it.

\subsection{Route Discovery}

Route discovery consists of two sub-procedures: Route Request (RREQ) and Route Replay (RREP). Route discovery is the mechanism by which a node wishing to send a packet to a destination node finds a route. Route discovery is used only when a node attempts to send a packet to another node and does not already know a route to that node. [7]

- Let consider a node $\mathrm{S}$ which want to send packets to node D. S will check its "Route cache" if route is present. If no route is found, it will have to start a route discovery protocol to find a route to the destination. Else the cached route is used to send packet.

- Now node $\mathrm{S}$ will initiate Route Request and will broadcast RouteRequest (RREQ) packet with a unique identification number. This RREQ is flooded throughout the network. Each node on receiving the RREQ packet rebroadcast the packet to neighbour nodes except some conditions like if the node has forwarded this packet already or if the node is destination node or time to live has exceeded. Each node before forwarding appends its own address to packet.

- Now how the nodes will check if it has forwarded the packet already? Solution to this is also given that each RREQ packet has a unique identification number generated by source node and path it has traversed. A node on receiving the RouteRequest packet checks the sequence number in routing table to check the duplicate request. Duplicate request is discarded.

\subsection{Route Maintenance}

Route maintenance is the mechanism by which node $\mathrm{S}$ is able to detect the change in network topology, such that if it is not able to use its route to $\mathrm{D}$ because a link along the route no longer works. Route maintenance is used only when $\mathrm{S}$ is actually sending packets to $\mathrm{D}$. When route maintenance indicates a source link is broken, $\mathrm{S}$ attempts to use any other route it know to $\mathrm{D}$, or invokes route discovery again to find a new route.

Using route discovery $S$ will get the RouteReply from D. Now when there is a path from $S$ to $D$ and $S$ can send packets to $D$ via selected route. Now when $S$ will send packets and if the intermediate node $\mathrm{C}$ moves from its position and cause wireless link breakage. RouteError (RERR) message will be sent by intermediate node $\mathrm{B}$ to initiator $\mathrm{S}$. Then source node $\mathrm{S}$ reinitiate route discovery for reestablishment of new route. The entries cached at intermediate nodes are removed when they get RERR message.

\section{PROBLEM FORMULATION}

Ad hoc networks are the autonomous systems consist of mobile nodes that communicate with each other using wireless communication. The highly dynamic natures of the mobile nodes create frequent and unpredictable network topology changes. These topology changes increase the routing complexity among the mobile nodes within the network. There for several traditional routing algorithms are not sufficient to the successful routing in Adhoc network. Routing in an Adhoc depends on many other factors including topology, selection of routers, and location of request initiator and specific underlying characteristics that could serve as a heuristic in finding the path quickly and efficiently. This makes the routing area perhaps the most active research area within the Adhoc domain.

Especially over the last few years, numerous routing protocols and algorithms have been proposed and their performance under various network environments and traffic conditions closely studied and compared.

In order to facilitate communication within an Adhoc network, an efficient routing protocol is required to discover routes between mobile nodes. As in ad hoc networks mobile devices are battery operated and the battery technology has not been improving rapidly. Therefore power consumption is likely to remain an issue in mobile wireless network routing.

Since communication between two nodes depend on other intermediate nodes as transmission range of nodes are not much capable to communicate directly. Lack of central administration in ad hoc network burden the nodes to maintain all routing information. Since nodes have limited energy they get out of energy which results in interruption in communication link and decrease network lifetime. The death of a small set of nodes because of depleted power might cause partitioning of the network result less network lifetime.

Conventional routing protocols do not consider the power during route discovery and route establishment. As there are many routing protocol we are studying Dynamic source routing protocol for this research work, which is an on demand routing protocol. DSR Dynamic source routing protocol do not take care of energy factor. So we need to develop a routing protocol that considers energy an important factor during route discovery and route establishment. So that lifetime of network can be increased and decrease packet loss and delay in transfer of packets.

Our main motive is to analyse several power aware routing algorithms such as Minimum Total Transmission Power Routing (MTPR), Minimum Battery Cost Routing (MBCR) and load balancing routing protocol. Then we need to develop an Energy Efficient Power Aware routing protocol that taking the basic idea of DSR Dynamic source routing protocol.

\section{PROPOSED DYNAMIC SOURCE ROUTING POWER AWARE SOURCE ROUTING (DSR-PSR)}

The primary objective of DSR-PSR is to select the path for the specified source to destination in such a way that intermediate nodes have higher power. So idea is instead of following minimum hop count method during the route discovery phase, select those path using nodes energy level.

Following are the steps taken for this

DSR-PSR Algorithm 
I. The Source Node which wants to send packet initializes the data transmission request.

II. First it checks the route cache for the particular destination; if destination found it uses that path to send data else route discovery process is initiated.

III. At the time of route discovery, a route request (RREQ) packet broad casted by the source. The header of the RREQ packet includes <source_id, destination_id>,

IV. Parameters on each node defined are Node Id, Battery Status B_S

Battery status is further divided into 3 categories:

If (Battery Status < 20\%)

Then Set $B \_S=1$

If $(20 \%<=$ Battery Status $<=60 \%)$

Then Set $B \_S=2$

If (Battery Status $<60 \%$ )

Then Set $B \_S=3$

Value of Battery status B_S is updated at each node according to it.

V. Parameters Concerned during Route Search

$T \_B \_S$ (Total Battery Status),WNs (number of weak nodes) and Node_IDs.

Now according to Battery status B_S Total battery status T_B_S is calculated

If $\left(\mathrm{B} \_\mathrm{Si}==3\right.$ )

Then T_B_S = T_B_S + 3

Else-if (B_Si $==2$ )

Then T_B_S = T_B_S + 1

Else-if $\left(\mathrm{B} \_\mathrm{Si}==1\right)$

$\mathrm{WN}=\mathrm{WN}+1$

Here WN represents a weak node which has the energy less than $20 \%$.

VI. After threshold time $\mathrm{T}$ destination node check out the paths and find the path with small number of week nodes.

VII. Now the path taken out is the path with nodes having more energy but still there needs to make little changes as if Length of path chosen using DSR-PSR is greater than DSR then older path will be taken into action to save time for sending packets.

If(hopcount(path(DSR-

PSR $))>$ hopcount(path(DSR)))

Then

Use path(DSR)

\section{SIMULATION AND RESULTS}

This section presents the simulation environment, metrics and the results of the proposed DSR-PSR routing protocol. Simulation is done using Network Simulator NS2.25.

\subsection{Simulation Environment}

An Adhoc network of 50 nodes with a simulation time of 100 seconds to 1150 seconds for different scenario is considered. The mobile nodes were placed on a 1200 X 1200 flat grid. DSR is used as the routing protocols. Thus, 50 different nodes are involved in the communication. The mobility of the nodes depends on the Random Way Point Model. Transmission Range of each node is $250 \mathrm{~m}$. Receiving power of each node is $0.395 \mathrm{~W}$. Transmitting Power is $0.660 \mathrm{~W}$.

Following tables shows the parameters and values of parameters used in simulation.
Table1 Simulation Parameters

\begin{tabular}{|c|c|c|}
\hline & PARAMETERS & VALUE \\
\hline $\mathbf{1}$ & Network Size & $1200 \mathrm{X} 1200$ \\
\hline $\mathbf{2}$ & No. of Nodes & 50 \\
\hline $\mathbf{3}$ & Simulation Time & $\begin{array}{c}350 \text { Sec-1150 Sec for } \\
\text { different scenario }\end{array}$ \\
\hline $\mathbf{4}$ & Traffic Type & $\begin{array}{c}\text { Constant Bit Rate } \\
\text { (CBR) }\end{array}$ \\
\hline $\mathbf{5}$ & Queue Type & Drop Tail \\
\hline $\mathbf{6}$ & Propagation Model & TwoRayGround \\
\hline $\mathbf{7}$ & Packet Size & 512 byte \\
\hline $\mathbf{8}$ & rxPower & 0.395 W \\
\hline $\mathbf{9}$ & txPower & 0.660 W \\
\hline $\mathbf{1 0}$ & MAC Protocol & 802.11 \\
\hline $\mathbf{1 1}$ & Mobility Model & Random Waypoint \\
\hline $\mathbf{1 2}$ & Routing Protocol & DSR \\
\hline $\mathbf{1 3}$ & Observation & $\begin{array}{c}\text { First Dead Node, } \\
\text { Percentage of all Dead } \\
\text { nodes, Throughput }\end{array}$ \\
& Parameters & \\
\hline
\end{tabular}

\subsection{Simulation Metrics}

DSR and DSR-PSR are compared using three simulation metrics those are:

\subsubsection{First dead node}

Comparing DSR with new proposed DSR-PSR simulation results shows that the first dead node in DSR-PSR comes later than DSR.

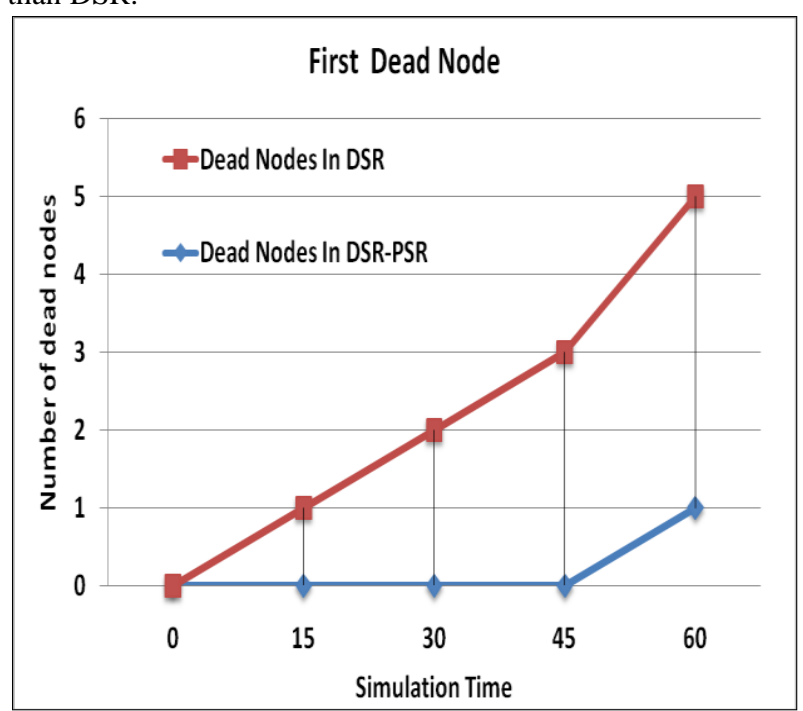

Fig 2 Comparison of First dead node in DSR and DSRPSR

\subsubsection{Percentage of Dead Nodes}

Both the protocol DSR and DSR-PSR are compared by percentage of dead nodes during specified interval of time. It is found that when $98 \%$ of nodes in DSR were dead only $16 \%$ of DSR-PSR nodes were dead .this shows the efficiency of DSR-PSR over DSR. 


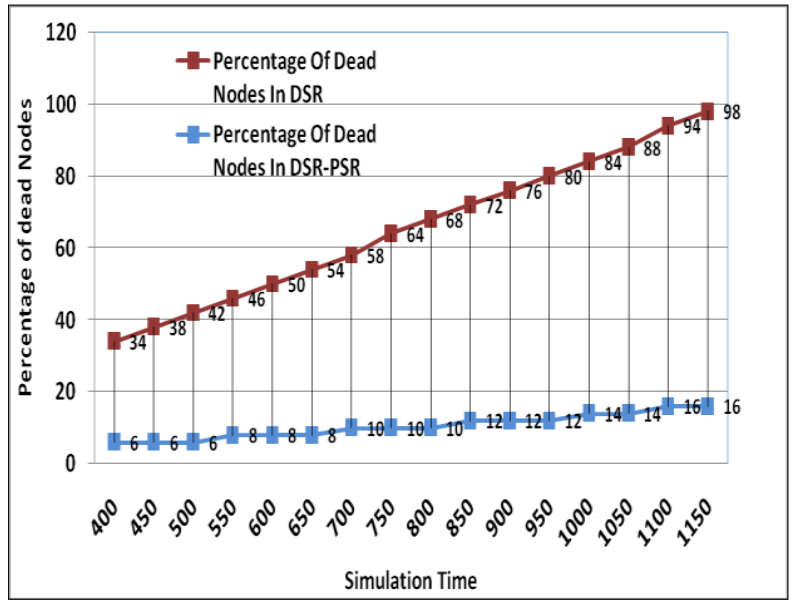

Fig 3 Comparison of percentage of dead nodes in DSR and DSR-PSR

\subsubsection{Throughput}

Throughput is defined as number of packets sent per unit of time. Comparing throughput we find that number of packets send per unit time in DSR-PSR is more than that of DSR.

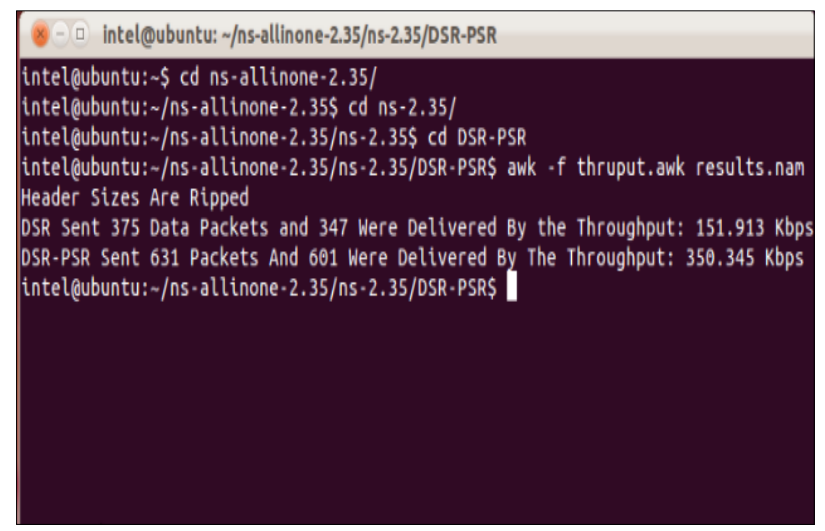

Fig 4 Throughput of DSR and DSR-PSR

\section{CONCLUSION}

The proposed protocol "DYNAMIC SOURCE ROUTING POWER AWARE SOURCE ROUTING" introduces power awareness into the routing decisions and finds path from source to destination.

- The best optimal path is chosen from the different path received by destination with in threshold time. The optimality of path is chosen on the energy factor. DSRPSR is being compared with DSR (dynamic source routing protocol) which do not consider energy during path discovery.

- Proposed algorithm provides a significant increase in successful packet transmissions i.e. high throughput and less number of dead nodes as it considers energy during path selection, so nodes with less energy are not taken into account.

Also as if in some cases the hop count in DSR-PSR is more than in DSR it will consider the older DSR algorithm to find path which saves the time.

\section{FUTURE WORK}

Future perspective of this research is as we have just introduced route discovery phase in it. Route management for broken links can be introduced. Also rather than choosing the simple shortest path first with energy factor we can use swarm optimization technique like ant colony optimization with primary factor energy to find optimal path from source to destination.

\section{REFERENCES}

[1] Subir Kumar Sarkar, T G Basavaraju, C Puttamadappa (2008) "Ad hoc mobile wireless networks principles, protocols and applications" Auerbach Publications Taylor \& Francis Group (ISBN 978-1-4200-6221-2)

[2] Wong, K.D. Kwon, T.J. Varma, (2004) "Towards commercialization of ad hoc networks", IEEE International Conference, Volume: 2

[3] Wikipedia Free multi language on-line encyclopedia, Retrieve on 12th February 2013 from the World Wide Web

[4] http://en.wikipedia.org/wiki/Wireless_ad_hoc_network

[5] http://en.wikipedia.org/wiki/Routing

[6] Magnus Frodigh, Per Johansson, Peter Larsson (2000) "Wireless ad hoc networking-the art of networking without a network",

[7] Imrich Chlamtac, Marco Conti, Jennifer J.-N. Liu "Mobile ad hoc networking: imperatives and challenges" , Elsevier 2003

[8] Ram Ramanathan and Jason Redi; "A brief overview of ad hoc networks: Challenges and directions", BBN Technologies, IEEE Communications Magazine 50th Anniversary Commemorative Issue May 2002

[9] C. Siva Ram Murthy, B.S. Manoj "Ad Hoc Wireless Networks: Architectures and Protocols" Pearson Education, Inc. (ISBN 978-0-1330-0706-0) September 2004

[10] IETF Mobile Ad-hoc Networks (manet), The Internet Engineering Task Force (IETF), provides a selected rfc related to MANETs networks http://www.ietf.org/ The Dynamic Source Routing Protocol (DSR) February 2007 http://www.ietf.org/rfc/rfc4728.txt

[11] RFC 3626 (OLSR) Optimized Link State Routing October 2003 http://www.ietf.org/rfc/rfc3626.txt

[12] Fisheye State Routing Protocol (FSR) for Ad Hoc Networks June 17, 2002 http://tools.ietf.org/html/draftietf-manet-fsr-03

[13] Ad hoc On-Demand Distance Vector (AODV) Routing July 2003 http://www.ietf.org/rfc/rfc3561.txt

[14] The Dynamic Source Routing Protocol (DSR) February 2007 http://www.ietf.org/rfc/rfc4728.txt

[15] S. Chettibi and m. Benmohamed, "A multipath energyaware on demand source routing protocol for mobile adhoc networks" computer sciences dpt., university mentouri of constantine, algeria. 2009

[16] Meng Li, Lin Zhang, Victor O. K. Li, Xiuming Shan, Yong Ren , "Energy-aware multipath routing protocol for mobile ad hoc networks" Department of Electronic 
Engineering Tsinghua university, ACM Sigcomm Asia'05, Apr. 10-12, 2005, Beijing, China

[17] Morteza Maleki, Karthik Dantu, and Massoud Pedram, "Power-aware source routing protocol for mobile ad hoc networks" Dept. Of ee-systems, University of Southern California, Los Angeles IEEE conference 2002 page $72-75$

[18] Giampaolo Bella, Gianpiero Costantino, Jon Ccrowcroft, "Enhancing DSR maintenance with power awareness" Salvatore Riccobene department of mathematic and Informatics, Italy 2012 Elsevier B.V.

[19] Carla F. Chiasserini, Ramesh R. Rao "Routing protocols to maximize battery efficiency" Department of electronics, Italy MILCOM 2000. 21st Century Military Communications Conference Proceedings page 496-500

[20] Ram Ramanathan, Regina Rosales-Hain, "Topology Control of Multihop Wireless Networks using Transmit Power Adjustment" Internetwork Research Department BBN Technologies (A Division of GTE) Cambridge, Massachusetts IEEE 2000

[21] Jae-Hwan Chang and Leandros Tassiulas "Energy Conserving Routing in Wireless Ad-hoc Networks" IEEE 2000

[22] Vinay Rishiwal, Mano Yadav, S. Verma, S. K. Bajapai "Power Aware Routing in Ad Hoc Wireless Networks" JCS\&T Vol. 9 No. 2 October 2009 Page 101-109
[23] Weifa Liang, Yang Yuansheng Department of Computer Science, "Maximizing Battery Life Routing in Wireless Ad Hoc Networks" IEEE 2004

[24] S. Singh and C. Raghavendra, "PAMAS: Power-aware Multiple Access Protocol with Signaling for Ad Hoc Networks," ACM Computer Communication Review, July 1998 page $181-190$

[25] Baisakh ,Nileshkumar R Patel, Shishir Kumar "Energy Conscious DSR in MANET" 2nd IEEE International Conference on Parallel, Distributed and Grid Computing 2012 page $784-789$

[26] Anil kumar Kulkarni, Suresh Chimkode "Traffic Sensitive Dynamic Source Routing Protocol for Mobile Ad hoc Networks" International Journal of Computer Applications (0975 - 8887) Volume 51- No.16, August 2012

[27] Q. Qi and C. Chakrabarti "Improving the Battery Performance of Ad-Hoc Routing Protocols" IEEE 2005

[28] Kavita Sharma and Vivek Sharma "Energy Efficient Multipath Dynamic source routing protocol-A survay" International Journal of Advanced Research in Computer Science and Software Engineering Volume 3, Issue 5, May 2013 page 466-476 